\title{
Structural Implications of Interfacial Hydrogen Bonding in Hydrated Wy-Montmorillonite Clay
}

\author{
Nithya Subramanian ${ }^{1}$, Michael Whittaker $^{1}$, Colin Ophus $^{2}$, and Laura Lammers ${ }^{1,3}$ \\ ${ }^{1}$ Energy Geosciences Division, Lawrence Berkeley National Laboratory, Berkeley, CA, USA; \\ ${ }^{2}$ National Center for Electron Microscopy, Molecular Foundry, Lawrence Berkeley National Laboratory, \\ Berkeley, CA, USA; \\ ${ }^{3}$ Environmental Science, Policy and Management Department, UC-Berkeley, Berkeley, CA, USA
}

\section{Supplementary Information}

\section{Simulation Methods}

The LAMMPS package ${ }^{1}$ was used for all molecular dynamics simulations. We created the cis-vacant MMT layer with the crystallographic information in Table S1. The atoms were mirrored about the $\mathrm{x}-\mathrm{y}$ plane centered on the $\mathrm{Al}$ atoms and the mirror image atoms were flipped and translated by $-a / 3$, and the hydroxyl bond was rotated by $60^{\circ}$ about the z-axis. The unit cell was repeated to obtain a layer of size $40.65 \AA$ × $44.92 \AA$ (see Fig. S1 and CisVMMT.xyz file attached). The octahedral $\mathrm{Al}$ atoms were substituted at random with $\mathrm{Mg}$ such that a net negative of charge $-0.57 e$ exists per unit cell of clay. The simulation volume $(82 \times 44.92 \times 110) \AA^{3}$ was created with five layers of clay stacked on top of each other with basal spacings corresponding to the hydration state in the interlayers (Fig. S1 (c)). Periodic boundary conditions were imposed to the simulation volume along all three dimensions, thus rendering the clay particle infinite in the $y$ and finite along the $x$ direction. The boundary conditions also give rise to infinite number of clay aggregates along the $z$ direction, each of the five MMT layers separated by nanopores large enough to avoid interactions between aggregates. This assumption allows to mitigate the effect of tactoid/aggregate size from the results based on the conclusions of Tester et al ${ }^{2}$. The pairwise Lennard Jones energy was calculated using $E_{\text {pair }}=$ $4 \epsilon\left[\left(\frac{\sigma}{r}\right)^{12}-\left(\frac{\sigma}{r}\right)^{6}\right]$, where $r$ is the distance between two particles, and $\epsilon$ and $\sigma$ are the maximum depth of the potential energy well and the distance of zero potential, respectively. Lennard-Jones interaction terms between unsimilar atom types are calculated via Lorentz-Berthelot mixing rules. We employ a cutoff distance of $15 \AA$ for short-range interactions, and Ewald's summation method with an accuracy of $99.99 \%$ for long range electrostatics. A timestep of $1 \mathrm{fs}$ is used in all our simulations, and density profiles/maps are constructed with data acquired every $100 \mathrm{ps}$ and averaged over $5 \mathrm{~ns}$ of equilibration.

Edges of an MMT layer exhibit consist of highly reactive proton-dissociable silanol and aluminol groups yielding $\mathrm{pH}$ dependent charge ${ }^{3-5}$. The edge sites also serve as the boundary at which diffusion occurs between interlayers and the bulk or nanopore fluid. Studies have attempted to construct an accurate edge structure for tv pyrophyllite-like clays in the context of acid-base reactivity through conceptual models ${ }^{6}$ and more recently, through $a b$ initio models ${ }^{7}$. Owing to a lack of symmetry about $b$ (see Fig.1), cv MMT edges site configurations are different for the edges perpendicular to the [010] and [0피 crystallographic directions. Cleaving the B \& AC edges ${ }^{7}$ results in the dissociation of several siloxane bonds and a final healed edge structure that is energetically unfavorable. For the purposes of the current study, our attention was restricted to making the edge unreactive. Therefore, we employed $\mathrm{OH}$, and $\mathrm{OH}_{2}$ terminations to the edges of finite MMT layers to ensure a neutral edge charge (e.g. at point of zero net proton charge). We will perform $a b$ initio MD in combination with high-resolution imaging as part of future work to determine the diverse and realistic edge configurations for cv MMT. 
Table S1: Atomic parameters for cv MMT to construct the unit cell. The atoms are mirror about the $x$-y plane centered on Al and shifted along the $x$-axis by -a/3. The orientation of the hydroxyl bond is changed by $60^{\circ}$ to obtain the cv MMT $1 x$ unit cell.

\begin{tabular}{|c|c|c|c|}
\hline Space group & $C 121(5)$ & & \\
\hline Cell parameters & $\mathrm{a}=5.1800 \AA \mathrm{A}=8.9700 \AA \mathrm{c}=10.0700 \AA \mathrm{\AA} \beta=99.5000^{\circ}$ \\
\hline \multicolumn{4}{|c|}{ Atomic parameters } \\
\hline Atom & $\mathrm{x} / \mathrm{a}$ & $\mathrm{y} / \mathrm{b}$ & $\mathrm{z} / \mathrm{c}$ \\
\hline $\mathrm{Al} 1$ & 0 & 0 & 0 \\
\hline $\mathrm{Al} 3$ & 0 & 0.654 & 0 \\
\hline $\mathrm{Si} 1$ & 0.432 & 0.333 & 0.27 \\
\hline $\mathrm{Si} 2$ & 0.432 & 0.662 & 0.27 \\
\hline O1 & 0.489 & 0.496 & 0.335 \\
\hline $\mathrm{O} 2$ & 0.173 & 0.725 & 0.335 \\
\hline O3 & 0.17 & 0.268 & 0.32 \\
\hline $\mathrm{O} 4$ & 0.334 & -0.024 & 0.105 \\
\hline $\mathrm{O} 5$ & 0.417 & 0.656 & 0.109 \\
\hline $\mathrm{O} 6$ & 0.343 & 0.347 & 0.109 \\
\hline $\mathrm{H}$ & 0.934 & -0.624 & 0.105 \\
\hline
\end{tabular}




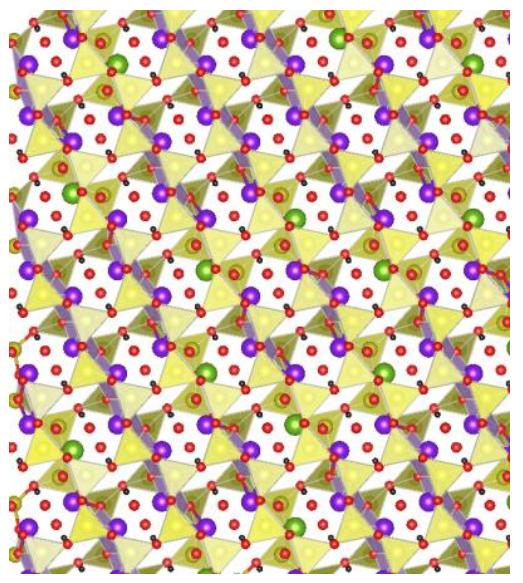

(a)

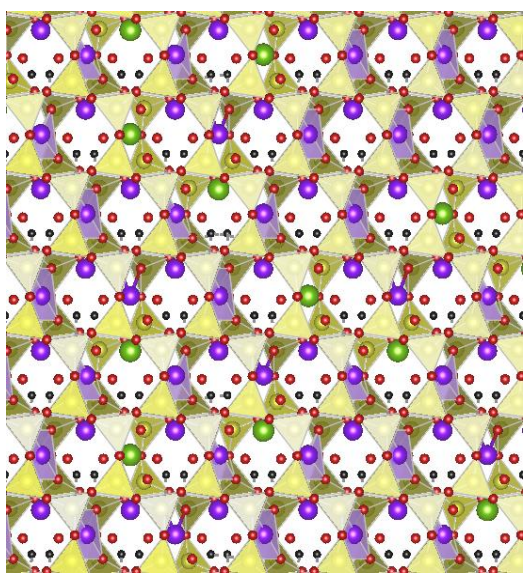

(b)

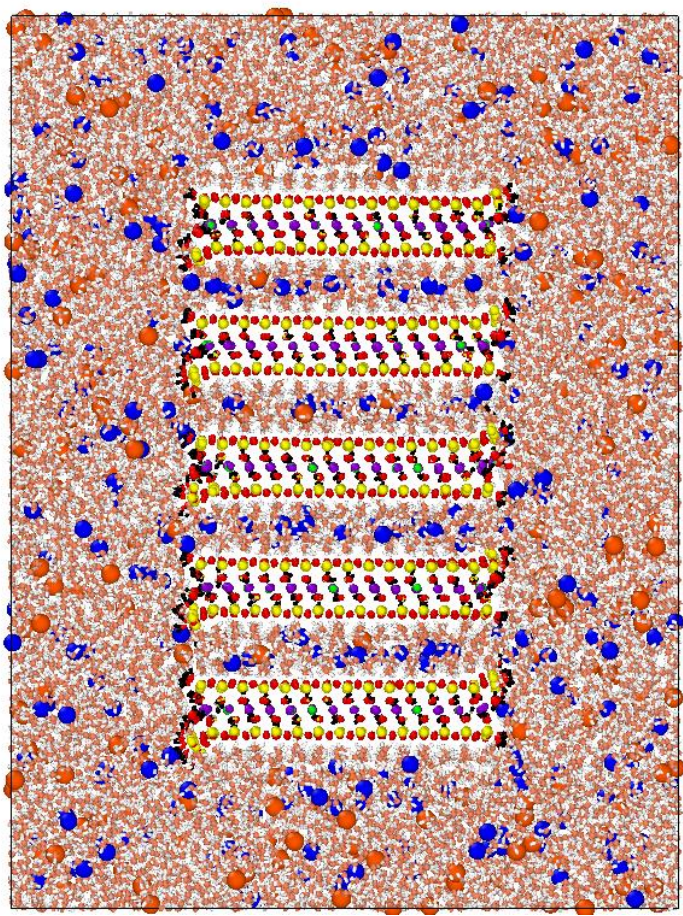

(c)

Figure S1: Molecular models of (a) Trans-vacant clay with hydroxyl groups occupying opposite ends of the vacancy; (b) New cisvacant clay structure based on HRTEM with hydroxyl groups in adjacent positions of the vacancy; (c) Simulation volume containing five MMT layers in bulk solution of $\mathrm{NaCl}$. Yellow corresponds to the Si tetrahedra, purple atoms are Al and corresponding octahedra; green atoms are random Mg substitutions; red atoms are clay oxygen and black are clay hydrogen. Bulk solution is shown with water oxygen atoms in pink, water hydrogen atoms in white, $\mathrm{Na}+$ ions in blue and Cl-ions in orange. 


\section{Calculation of Tetrahedricity}

To investigate the structure of water in the interlayer and the existence of order/symmetry, we used a quantitative measure called tetrahedricity, which characterizes the tetrahedral arrangement of water. The tetrahedricity parameter was calculated based on Equation-S1.

$$
\Theta=\frac{6 \sum_{j \neq i}\left(l_{i}-l_{j}\right)^{2}}{15 \sum_{i}\left(l_{i}\right)^{2}}
$$

Where $l_{i}$ corresponds to the length of the six edges of the tetrahedron formed by the four nearest neighbors of each water molecule. For an ideal tetrahedral arrangement, $\Theta$ is equal to zero. The tetrahedricity measure, thus, provides the deviation of an arrangement from an ideal one and is valuable for distinguishing between bulk water and 'ice-like' liquid. Figure S2 illustrates the distribution of the tetrahedricity measure in interlayer water on tv and cv clay. It is clear that the tetrahedricity parameter of water in cv MMT interlayers is closer to zero with a sharp peak centered at 0.02; this confirms that the interlayer water in the cv mineral configuration has a more 'ice-like' structure.

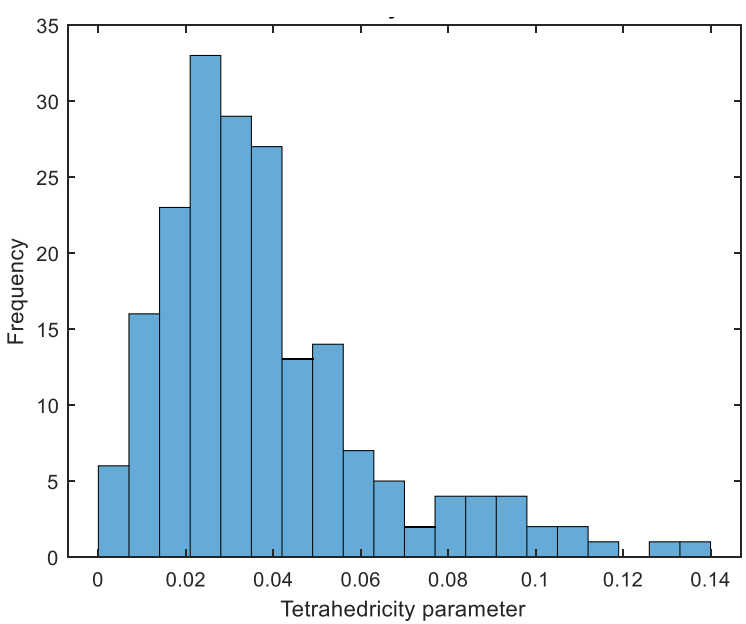

(a)

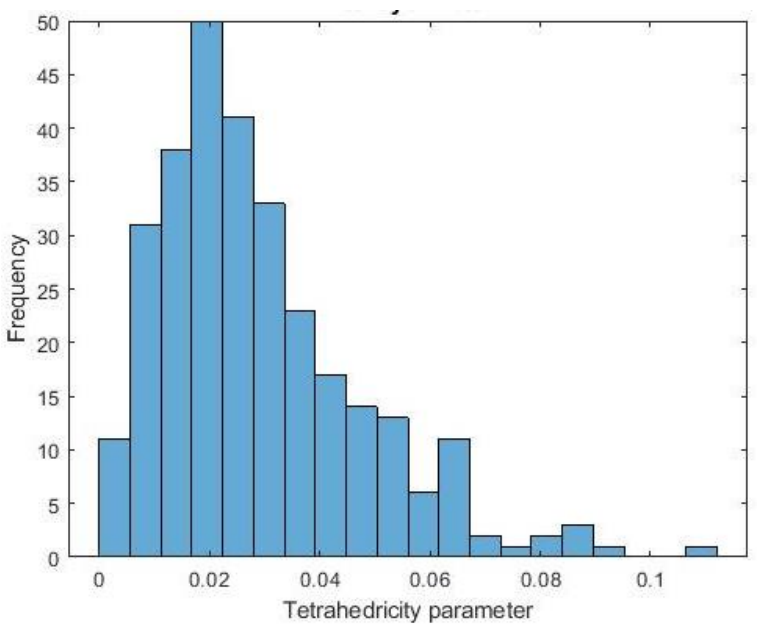

(b)

Figure S2: Tetrahedricity parameter of interlayer water in (a) trans-vacant clay; (b) cis-vacant clay. The peak of the distribution of tetrahedricity parameter is closer to zero in cv clay indicating that the interlayer water in cv clay is more 'ice-like'.

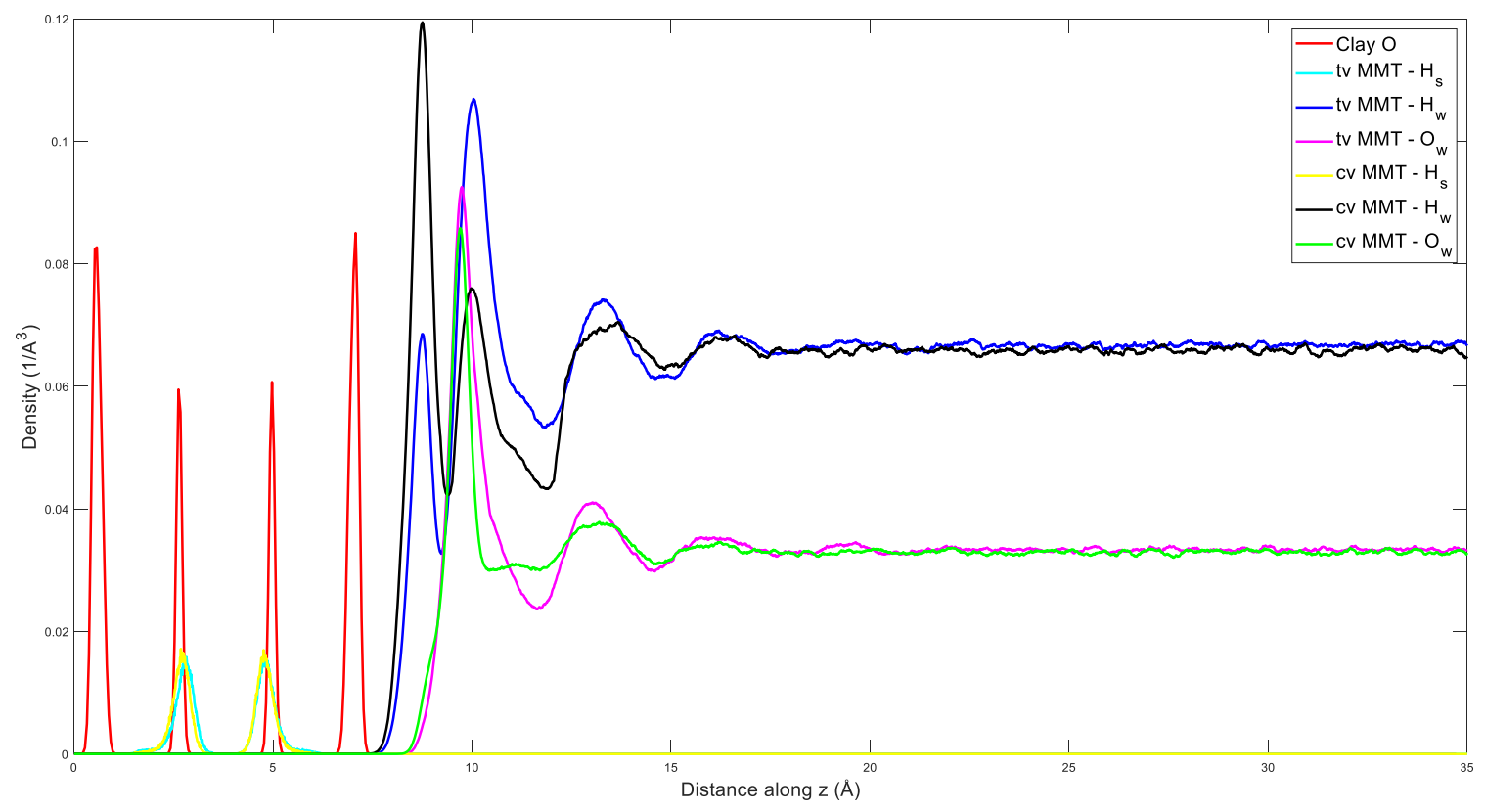

Figure S3: water oxygen and hydrogen density as a function of distance from tv and cv mineral surface 
Figure S3 corresponds to the detailed density profiles of structural oxygen and hydrogen atoms in MMT and the water hydrogen and oxygen atoms obtained from the $6.5 \mathrm{~nm}$ nanopore simulations described in Section 4.2 of the manuscript. The first $\mathrm{H}_{\mathrm{w}}$ peak of cv MMT has a much higher intensity indicating the presence of more water-H atoms pointing towards the $\mathrm{cv}$ mineral surface. A proportion of these $\mathrm{H}_{\mathrm{w}}$ are hydrogen bonded to the $\mathrm{Si}-\mathrm{O}-\mathrm{Si}$ oxygen atoms $(\approx 2 \AA)$, whereas the others are weakly hydrogen bonded to the hydroxyl groups $(\approx 3.7 \AA)$. Based on Figure 4 and Figure S3, we infer that water on the cv MMT surface is more likely to be doubly hydrogen-bonded to the surface. On the other hand, the second water-H peak on the tv MMT surface has a higher intensity suggesting the presence of stronger hydrogen bonding within the interlayer/bulk water. The structural $\mathrm{OH}$ bonds $\left(\mathrm{H}_{\mathrm{s}}\right)$ are pointing away from the interlayer in both tv and cv MMT (offset between $\mathrm{H}_{\mathrm{s}}$ and clayO).

\section{Experimental Methods}

Wyoming montmorillonite (SWy-2), obtained from the Source Clays Repository of The Clay Minerals Society (http://www.clays.org/sourceclays_data.html), was used throughout this study. An aqueous diluent phase, (herein called clay-saturated aqueous phase, CSAP) saturated with respect to $\mathrm{SWy}-2$, was made by dialyzing SWy-2 (10 mg) against MilliQ water $(1 \mathrm{~L}$, resistivity $=18.2 \mathrm{M} \Omega \mathrm{cm})$ for 1 week and filtering through a $0.02 \mu \mathrm{m}$ filter (Whatman). To obtain homoionic MMT, SWy-2 (2-10 g) was dispersed in $\mathrm{NaCl}$ or $\mathrm{KCl}(1 \mathrm{M}, 50 \mathrm{~mL}$ made from CSAP) in a polypropylene bottle $(50$ or $1000 \mathrm{~mL})$ and mixed on a rotating mixer $(5 \mathrm{rpm})$ for seven days. Sedimentation of coarse particles was achieved by centrifugation (1000 rcf for 10 minutes). Fine particles were separated from the coarse sediment and transferred to a cellulose dialysis membrane (Spectra/Por 3, $3.5 \mathrm{kDa}$ molecular weight cutoff). Excess salt in the supernatant was removed by dialysis against CSAP, which was replaced every day for seven days. The resulting clay gel was dried in a convection oven $\left(60^{\circ} \mathrm{C}\right.$ for 14 hours, then $110^{\circ} \mathrm{C}$ for 6 hours).

Suspensions were prepared by redispersing homoionic powder in CSAP to a final concentration of $5 \mathrm{mg} / \mathrm{mL}$ and sonicating in an ultrasonic bath at $30^{\circ} \mathrm{C}$ for 60 minutes. $1 \mathrm{~mL}$ of this solution was diluted $10 \times$ with CSAP and sonicated again for 60 minutes. Dilution and sonication was repeated again, for a total dilution of $100 \times$ (target concentration $0.05 \mathrm{mg} / \mathrm{mL}$ ). An aliquot of $3 \mu \mathrm{L}$ was pipetted into a lacy carbon TEM grid that had been glow discharged in air plasma for 60s immediately prior to applying sample. The sample was dried overnight in a convection oven at $110^{\circ} \mathrm{C}$ and transferred directly to the microscope for imaging.

Imaging was performed at the National Center for Electron Microscopy at Lawrence Berkeley National Laboratory on the TEAM 0.5 microscope operated in low-dose aberration corrected HRTEM mode at $300 \mathrm{kV}$. Spherical aberration was set to $-6 \mu \mathrm{m}$. A source monochromator was used to maintain the total dose below $500 \mathrm{e}^{-} / \AA^{2}$ at a dose rate of $121 \mathrm{e}^{-} / \AA^{2}$. Twenty-one dose was fractionated images with individual doses of $\sim 23 \mathrm{e}^{-} / \AA^{2}$ were aligned, background subtracted, and denoised with BM3D using custom software written in Matlab. Detector readout noise was filtered in Fourier space.

The crystal structure of bulk MMT powder was investigated by powder X-ray diffraction (pXRD) on a Rigaku diffractometer using $\mathrm{Cu} \mathrm{K} \alpha$ radiation $(\lambda=1.5404 \AA$. The (020) in-plane diffraction peak is clearly resolved in all samples at $q=1.40 \AA^{-1}(d=0.45 \mathrm{~nm})$ and is diagnostic of 2:1 layer phyllosilicates. ${ }^{1}$ Quartz was a major secondary phase, exhibiting peaks at $q=1.47,1.87$, and $2.5 \AA^{-1}$, labeled with (Q). 

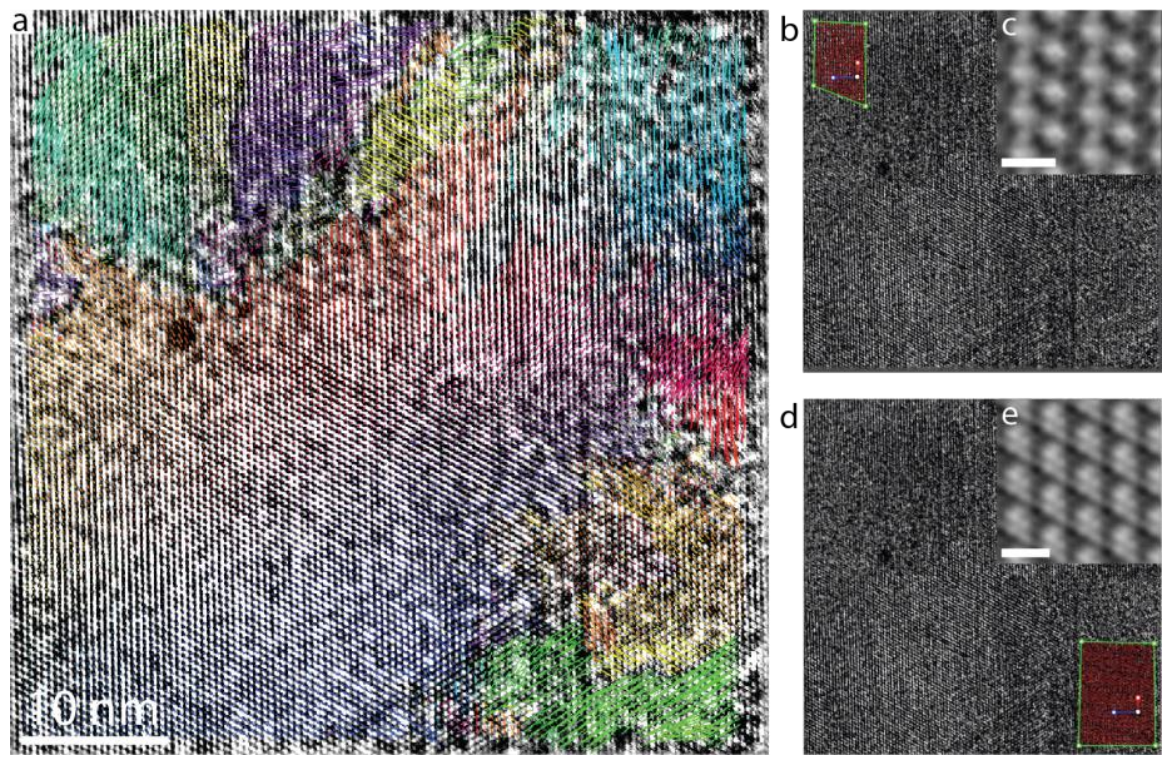

Figure S4: Lattice fitting of cv MMT crystal. (a) Map of unit cell displacement for cis-vacant MMT crystal, showing a predominantly planar region bottom-left with much greater displacements to the top and right. Abrupt changes in displacement vectors are inconsistent with regions in which the crystallite orientation changes but are consistent with a single bent crystal. (b)(e) Average unit cells from bent regions of crystal, showing deviations from planar region but retention of the overall symmetry.
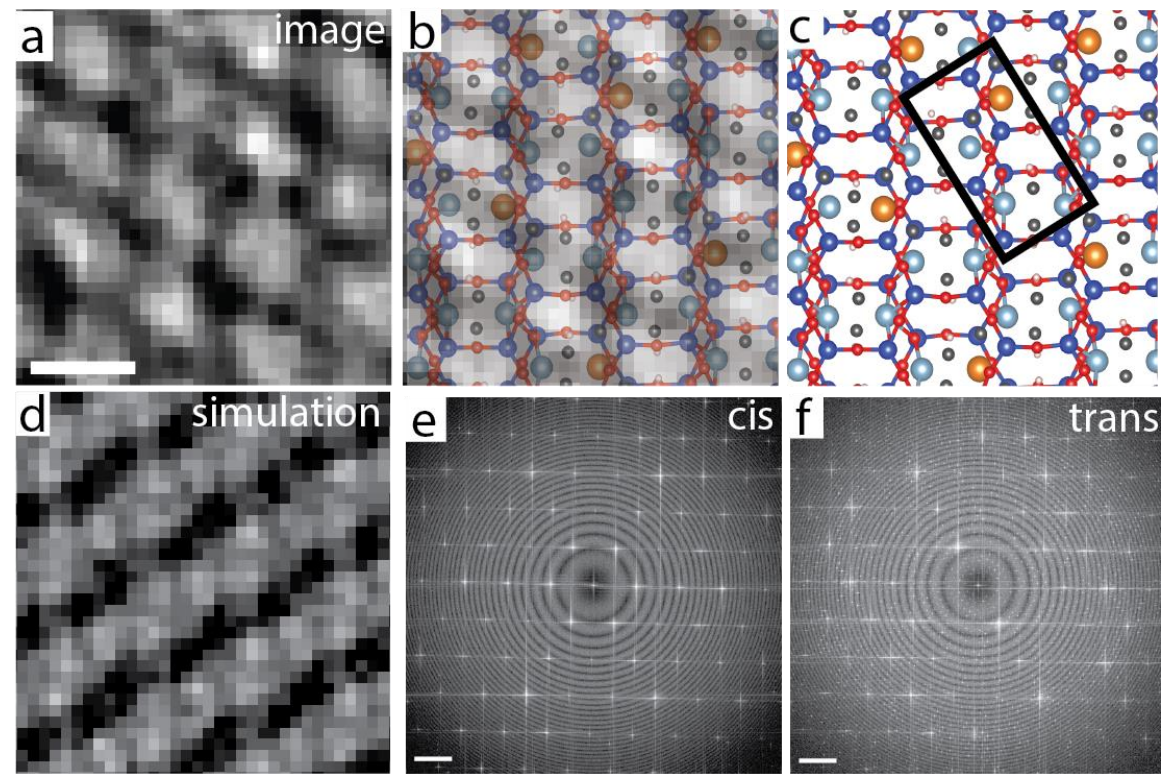

Figure S5: Comparison of cis- and trans-vacant structures. (a) Image from Fig. 2d; Scale bar: 5 A (b) Trans-vacant structure with overall orientation adopted from Fig. $2 b$ overlaid on Fig. S5-a. (c) Unit cell of trans-vacant structure. (d) Simulated image of transvacant structure in $(b)$ and $(c)$, with very poor correspondence to observed image. (e) Fourier transform of simulated cis-vacant structure from Fig. 2e, in good agreement with the experimental Fourier transform (Fig. 2b); (f) Fourier transform of trans-vacant image simulation $(d)$, with forbidden (110) and (110)-type spots, which were observed experimentally and thus preclude the possibility of a trans-vacant structure.

\section{References}

(1) Plimpton, S. Fast Parallel Algorithms for Short-Range Molecular Dynamics. J. Comput. Phys. 1995, 117 (1), 1-19.

(2) Tester, C. C.; Aloni, S.; Gilbert, B.; Banfield, J. F. Short- and Long-Range Attractive Forces That Influence the Structure of Montmorillonite Osmotic Hydrates. Langmuir 2016, 32 (46), 12039-12046.

https://doi.org/10.1021/acs.langmuir.6b03265. 
(3) Tournassat, C.; Davis, J. A.; Chiaberge, C.; Grangeon, S.; Bourg, I. C. Modeling the Acid-Base Properties of Montmorillonite Edge Surfaces. Environ. Sci. Technol. 2016, 50 (24), 13436-13445.

(4) Bourg, I. C.; Sposito, G.; Bourg, A. C. M. Modeling the Acid-Base Surface Chemistry of Montmorillonite. J. Colloid Interface Sci. 2007, 312 (2), 297-310. https://doi.org/10.1016/j.jcis.2007.03.062.

(5) Bourg, I. C.; Bourg, A. C.; Sposito, G. Modeling Diffusion and Adsorption in Compacted Bentonite: A Critical Review. J. Contam. Hydrol. 2003, 61 (1-4), 293-302.

(6) White, G. N.; Zelazny, L. Analysis and Implications of the Edge Structure of Dioctahedral Phyllosilicates. Clays Clay Miner. 1988, 36 (2), 141-146.

(7) Newton, A.; Kwon, K.; Cheong, D.-K. Edge Structure of Montmorillonite from Atomistic Simulations. Minerals 2016, 6 (2), 25. https://doi.org/10.3390/min6020025. 